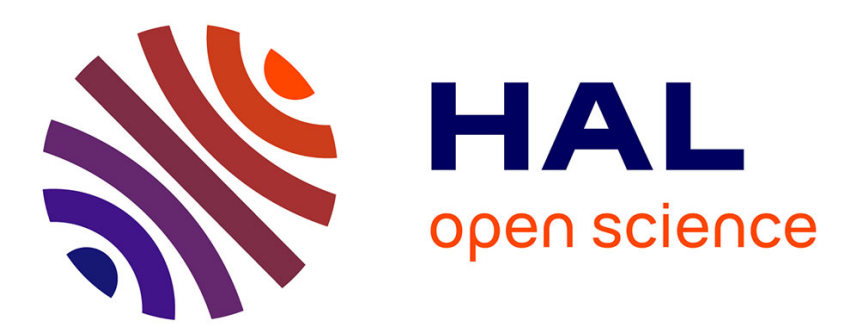

\title{
Fibrillation Patterns Creep and Jump in a Detailed Three-Dimensional Model of the Human Atria
}

\author{
Mark Potse, Alain Vinet, Ali Gharaviri, Simone Pezzuto
}

\section{To cite this version:}

Mark Potse, Alain Vinet, Ali Gharaviri, Simone Pezzuto. Fibrillation Patterns Creep and Jump in a Detailed Three-Dimensional Model of the Human Atria. FIMH 2019 - 10th International Conference Functionnal Imaging and Modeling of the Heart, Yves Coudière; Valéry Ozenne; Edward Vigmond; Nejib Zemzemi, Jun 2019, Bordeaux, France. pp.131-138, 10.1007/978-3-030-21949-9_15 . hal02153733

\section{HAL Id: hal-02153733 \\ https://hal.inria.fr/hal-02153733}

Submitted on 12 Jun 2019

HAL is a multi-disciplinary open access archive for the deposit and dissemination of scientific research documents, whether they are published or not. The documents may come from teaching and research institutions in France or abroad, or from public or private research centers.
L'archive ouverte pluridisciplinaire HAL, est destinée au dépôt et à la diffusion de documents scientifiques de niveau recherche, publiés ou non, émanant des établissements d'enseignement et de recherche français ou étrangers, des laboratoires publics ou privés. 


\title{
Fibrillation Patterns Creep and Jump in a Detailed Three-Dimensional Model of the Human Atria
}

\author{
Mark Potse $^{1,2,3}$, Alain Vinet ${ }^{4}$, \\ Ali Gharaviri ${ }^{5}$, and Simone Pezzuto ${ }^{5}$ \\ 1 Univ. Bordeaux, IMB, UMR 5251, Talence, France \\ 2 CARMEN Research Team, Inria Bordeaux Sud-Ouest, Talence, France \\ ${ }^{3}$ IHU Liryc, fondation Bordeaux Université, Pessac, France \\ mark@potse.nl \\ 4 Institut de génie biomédical, Université de Montréal, Montreal, Canada \\ alain.vinet@umontreal.ca \\ 5 Center for Computational Medicine in Cardiology, \\ Institute for Computational Science, \\ Università della Svizzera italiana, Lugano, Switzerland \\ ali.gharaviri@usi.ch, simone.pezzuto@usi.ch
}

abstract Activation mapping in animal models of atrial fibrillation (AF) has shown that activation patterns can repeat for several cycles and then be followed by different changing or repetitive patterns. Subsequent clinical studies have suggested a similar type of activity in human AF. Our purpose was to investigate whether a computer model of human AF can reproduce this behavior.

We used a three-dimensional model of the human atria consisting of $0.2-\mathrm{mm}$ volumetric elements with a detailed representation of bundle structures and fiber orientations. Propagating activation was simulated over 9 seconds with a monodomain reaction-diffusion model using human atrial membrane dynamics. AF was induced by rapid pacing. Pattern recurrence was quantified using a similarity measure based on transmembrane voltage at 1000 uniformly distributed points in the model.

Recurrence plots demonstrated a continuous evolution of patterns, but the speed of evolution varied considerably. Groups of upto 15 similar cycles could be recognized, separated by rapid changes. In a few cases truly periodic patterns developed, which were related to macroscopic anatomical reentry. The drivers of non-periodic patterns could not be clearly identified. For example, a spiral wave could appear or disappear without an obvious impact on the similarity between cycles.

We conclude that our model can indeed reproduce strong variations in similarity between subsequent cycles, but true periodicity only in case of anatomical reentry.

keywords Atrial fibrillation, Reentry, Recurrence plot, computer model

Published in: Y. Coudière et al. (Eds): Functional Imaging and Modeling of the Heart. Lecture Notes in Computer Science vol. 11504, pp. 131-138, Springer, 2019. doi : 10.1007/978-3-030-21949-9_15 


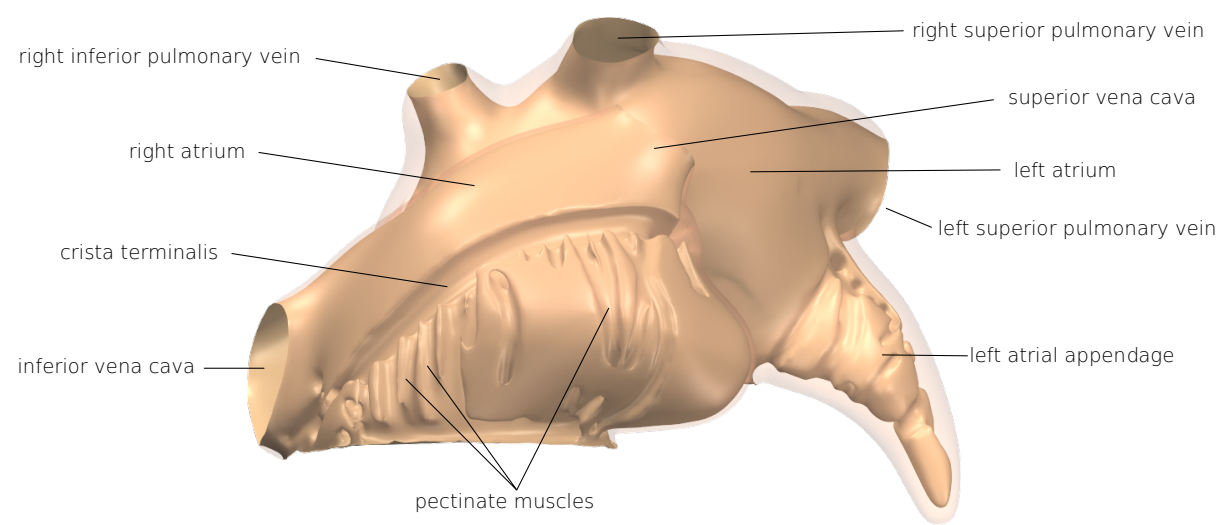

Fig. 1. Anatomy of the atrial model. The epicardium is rendered semi-transpararently to show the variations in wall thickness.

\section{Introduction}

Optical mapping of atrial fibrillation (AF) in sheep atria has shown that activation patterns can repeat for several cycles and then suddenly give way to a sequence of dissimilar cycles or another repetitive pattern [12]. Clinical studies have suggested a similar type of activity in human AF [8]. In the sheep atria these repetitive patterns were related to temporarily stable rotor activity. In human AF such a link is harder to establish, because AF patterns in patients can only be observed with coarse resolution by noninvasive mapping [8] or in a small portion of the atria by invasive mapping [1].

A computer model can help to gain more insight in this pseudo-stable behavior of AF, in its determinants, and in possible methods to recognize it with limited data, such as an ordinary electrocardiogram. The purpose of this study was therefore to investigate whether a computer model can reproduce the AF dynamics observed in animal studies, and if such behavior can be linked to meandering spiral waves.

\section{Methods}

A three-dimensional model of the human atria was previously constructed $[5,10]$. The model included the crista terminalis, pectinate muscles, Bachmann's bundle, posterior interatrial bundles, bundle structures in the left atrial appendage, and up to three layers of fiber orientation (figure 1).

Propagating activation was simulated with a monodomain reaction-diffusion model using human atrial membrane dynamics [2], with minor adaptation to avoid numerical instabilities due to discontinuous coefficients, on a hexahedral mesh with $0.2-\mathrm{mm}$ spacing. The model was integrated with the Rush-Larsen method for gating variables and an explicit Euler method for the other variables, using a fixed time step of $0.01 \mathrm{~ms}$. 
To simulate electrically remodeled atria we modified the maximum conductivities of the calcium current, $g_{\mathrm{CaL}}$, from 0.1238 to $0.037, g_{\mathrm{TO}}$ from 0.1652 to $0.026, g_{\mathrm{K} 1}$ from 0.09 to 0.180 , and $g_{\mathrm{Ks}}$ from 0.129 to $0.1 \mathrm{nS} / \mathrm{pF}$. [4].

Simulations were performed in a "fibrotic" model and in a "normal" model. In the fibrotic model $50 \%$ of the model elements had zero transverse conductivity, to mimic the uncoupling of lateral connections in ageing myocardium [13]. The affected elements were chosen randomly, with a uniform probability density.

With each model 10 simulations, each with 9 seconds duration, were performed. AF was induced by rapid pacing, from a different location in each simulation. Pattern recurrence and similarity between simulations were quantified at 1-ms intervals using a state variable consisting of transmembrane voltage sampled from 1000 distributed points in the model. The points were chosen randomly, regardless of whether they were endocardial, epicardial, or intramural. For each pair of time samples $i, j$, a similarity measure $S_{i j}$ was computed as

$$
S_{i j}=\left(\Phi_{i} \cdot \Psi_{j}\right) /\left(\left|\Phi_{i}\right| \cdot\left|\Psi_{j}\right|\right)
$$

where $\Phi_{i}$ is the state at time $i$ in one simulation and $\Psi_{j}$ the state at time $j$ in another; $\Phi \equiv \Psi$ for self-similarity or "recurrence." This similarity was visualized using recurrence plots [3,9], which show $S_{i j}$ as a function of $i$ and $j$.

Phase singularity filaments were identified using a phase measure computed with a time embedding of the transmembrane potential [6] with a delay of $10 \mathrm{~ms}$. On each face of the computational mesh, the topological charge was computed as the line integral of the phase over the corners. If the integral was $\pm 2 \pi$ a singularity filament was assumed to cross the face. Crossings that occurred on different faces of the same element were assumed to be part of the same filament.

Simulations were performed with the Propag-5 software [7] on 96 compute nodes each containing two 14-core Intel Xeon E5 processors. A full 9-second simulation took 55 minutes.

\section{Results}

\subsection{Normal model}

In the normal model, 3 out of 10 simulations yielded AF until the end of the simulation (9 seconds). In the other 7 activity stopped between 536 and $1398 \mathrm{~ms}$ after the last pacing pulse (approximately 4 to 9 cycles), either before any reentrant pattern had established or after all wavefronts encountered refractory tissue.

In the 3 simulations that yielded 9 seconds of $\mathrm{AF}$, periods of higher and lower similarity between subsequent cycles could be discerned. An example is shown in figure 2. Panel A shows $S_{i j}$ together with all phase singularity filaments that lasted more than $150 \mathrm{~ms}$. The first such filaments appear shortly before the end of the pacing interval. Subsequently, several intervals with simultaneous filaments are seen, separated (around 3.6 s) by a short interval without any long-living filaments. Panel B shows, for each time instant in the simulation, the similarity of the three best-matching time instants in the simulation separated by at least 
A

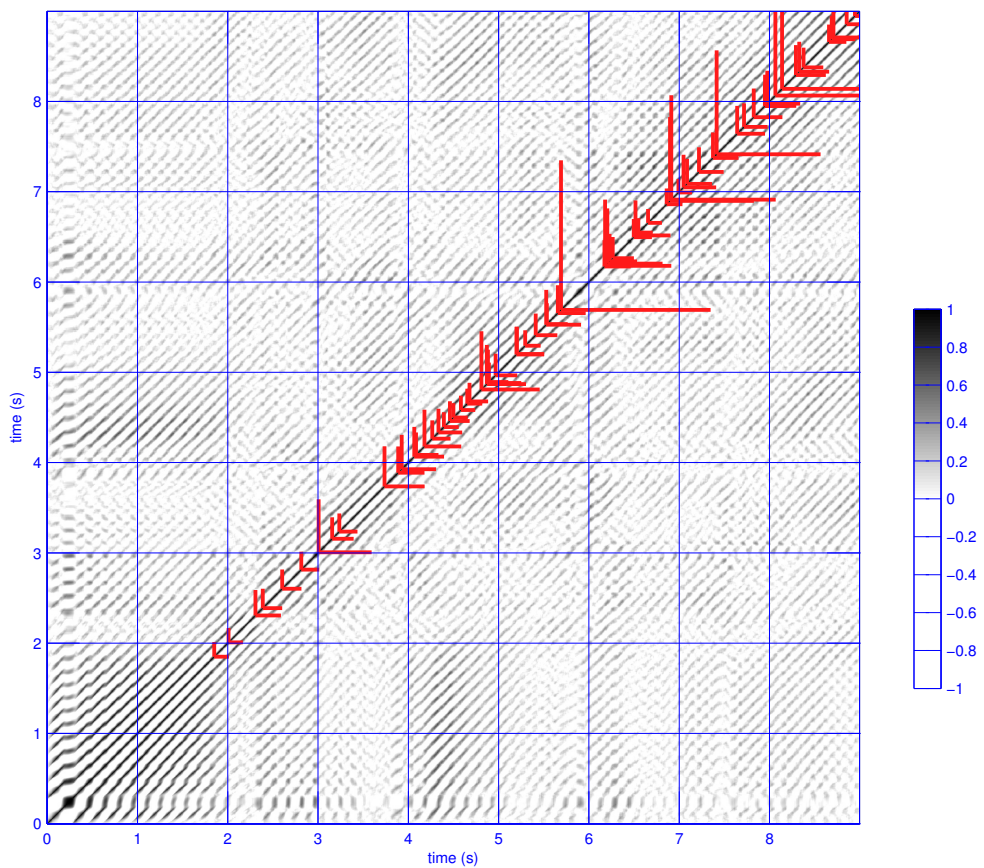

B

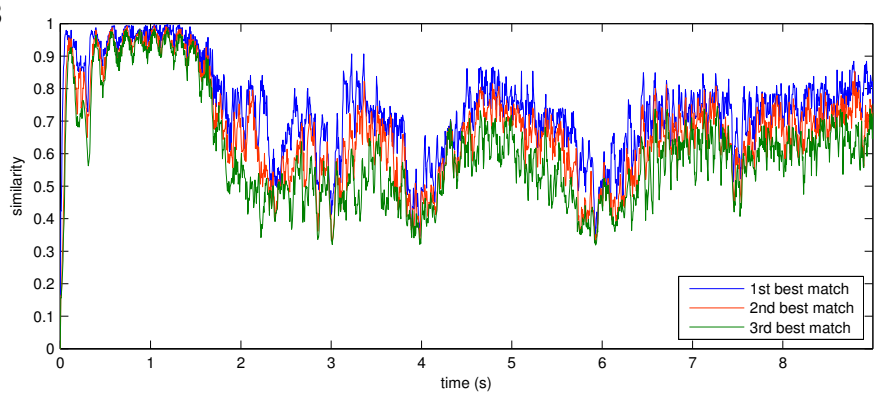

Fig. 2. A: Recurrence plot, showing $S_{i j}$ for a simulation in the normal model. Darker shades of gray represent higher similarity between the potential patterns at each pair of time instants. A dark diagonal line outside the main diagonal indicates a repeating activation pattern. Here, after 2 seconds of rapid pacing, periods of higher and lower similarity follow. Red lines indicate the intervals during which long-living phase singularity filaments were present. B: Graph showing the similarity of the three bestmatching time instants for each time instant in the simulation.

50 ms; i.e. $S_{i j_{1}}>S_{i j_{2}}>S_{i j_{3}}>\ldots$ where $\left|j_{n}-i\right|>50$ and $\left|j_{n}-j_{m}\right|>50$ for any $j_{n}, j_{m}$. Maximum similarity is seen to oscillate roughly between 0.4 and 0.8 . There is no obvious relation with periods of rotor activity.

In all 10 simulations with the normal model combined, 7940 separate singularity filaments were detected. 2324 of these $(29 \%)$ were detected for only a single time instant, 138 filaments lived longer than $150 \mathrm{~ms}$ (approximately one 
cycle length), and 68 lived longer than $300 \mathrm{~ms}$. The longest-living filament lasted for $5601 \mathrm{~ms}$.

\subsection{Fibrotic model}

In the fibrotic model, 5 out of 10 simulations yielded AF until the end of the simulation. In the other 5 , activity stopped between 655 and $1268 \mathrm{~ms}$ after the last pacing pulse.

As in the normal model, recurrence plots demonstrated a continuous evolution of patterns, with a varying speed of evolution. A "creeping" progression during groups of upto 15 similar cycles could be recognized, separated by rapid "jumps." An example is shown in figure 3.

In the fibrotic model, three of the 5 simulations that showed activity until the end of the simulation arrived at the same periodic regime, which corresponded to an anatomical reentry that passed from the coronary sinus to the left atrium and returned through the interatrial septum. A comparison of two of these simulations is shown in figure 4 .

In all 10 simulations with the fibrotic model combined, 20873 separate filaments were found. 7898 of these $(38 \%)$ were detected for only a single time instant, 31 filaments lived longer than $150 \mathrm{~ms}$, and 11 lived longer than $300 \mathrm{~ms}$. The longest-living filament lasted for $2901 \mathrm{~ms}$.

\section{Discussion}

This study shows that a computer model can reproduce variations in similarity between subsequent cycles of AF. The results also highlight that it is difficult to define when a pattern is "periodic," as it was called by Skanes et al. [12], because the similarity between subsequent cycles was never perfect, and ranged between 0.4 and 0.98 in our results. Since published studies have not quantitatively defined when patterns were considered periodic or nonperiodic, a quantitative comparison to their results was not attempted here.

In addition to the similarity of patterns between different time instants we also studied the occurrence of phase singularity or "vortex" filaments. Here we found surprising results. The total number of detected filaments was 2.5 times larger in the fibrotic model than in the normal model, while the total time spent in AF was only 1.6 times larger. In striking contrast, the number of filaments that lived longer than $150 \mathrm{~ms}$ was 5 times smaller in the fibrotic model, despite the longer time in AF. Clearly the presence of fibrosis in this model promotes the generation of phase singularities but also reduces their lifetime. Thus, if one were to use the number of phase singularities to demonstrate that fibrosis changes the complexity of AF, the result would greatly depend on the definition of a separate phase singularity.

A clear relation between nearly-periodic patterns and the existence of longlived phase singularities could not be established. This may be partly explained by methodological restrictions. For example, when a boundary between excitable 
A

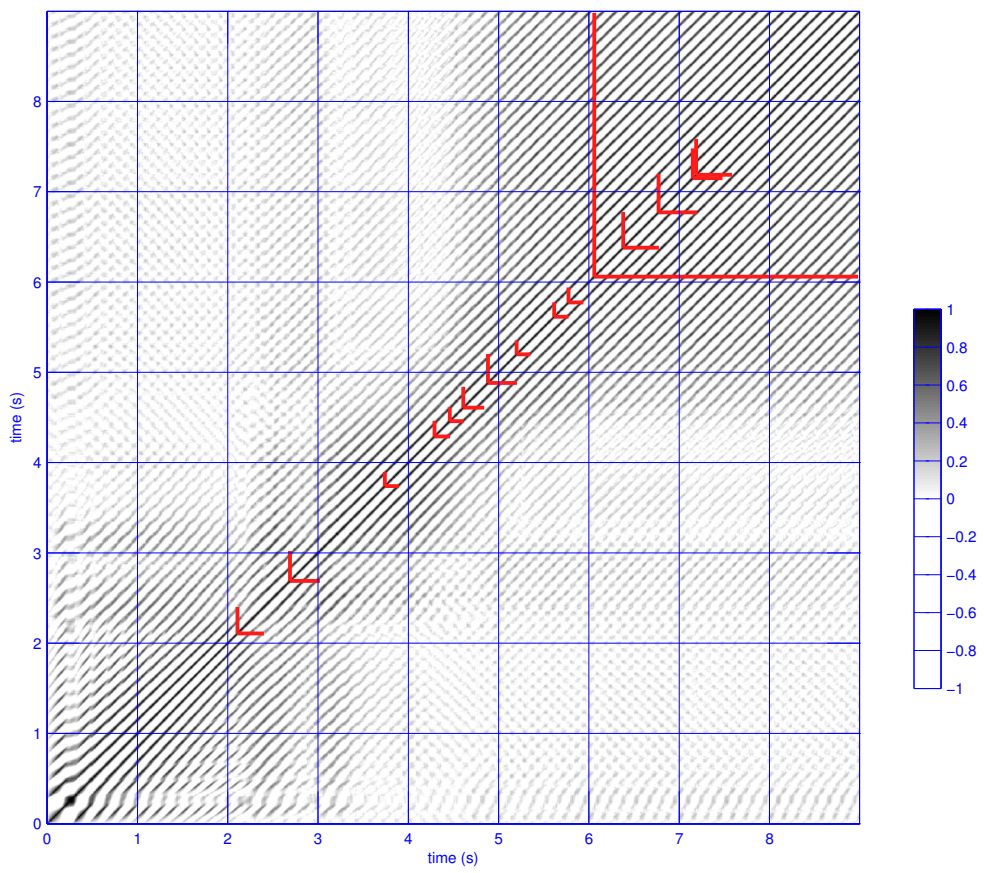

B

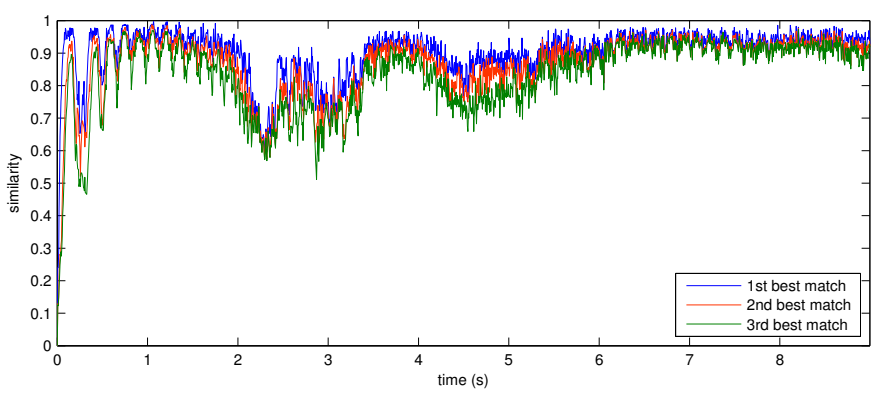

Fig. 3. A: Recurrence plot, showing $S_{i j}$ for a simulation in the fibrotic model. The format is as in figure 2. After 2 seconds of rapid pacing, the development of 3 groups of similar cycles can be recognized. Long-living phase singularities (red lines) are present but do not coincide with the groups of similar cycles. B: Similarity of the three bestmatching time instants for each time instant in the simulation.

and refractory tissue crosses an anatomical obstacle such as a pulmonary vein, a vortex filament traveling along this boundary may enter the obstacle and reappear on the other side. In such cases, our methods will see this as two different filaments. It is in general very difficult to determine the type of reentry that takes place in this complex anatomy. Similarly, the precise mechanism of termination of a reentry could not always be understood.

Our results concerning vortex filaments likely depend on the methods chosen to detect the intersections of filaments with mesh elements [14] and the deci- 


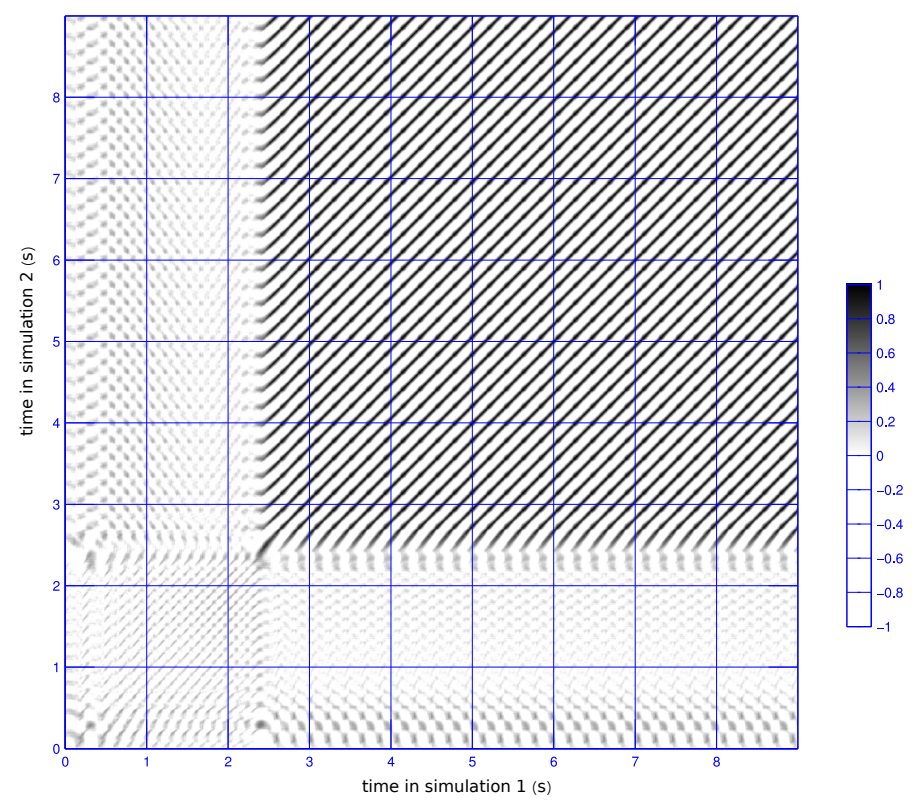

Fig. 4. Similarity plot for two simulations in the fibrotic model, which ended up in the same periodic regime. The format is the same as in figure 3 . The simulations were stimulated at different locations, leading to different patterns during the first $2.3 \mathrm{~s}$. Subsequently the two simulations produced exactly the same patterns, albeit with a phase delay (none of the lines of maximum intensity coincides exactly with the main diagonal).

sions whether different intersections belong to the same filament and whether filaments in subsequent time steps are related. A comparison of these methods, however, is outside the scope of this study. The type of fibrosis and the method to model it [11] will also affect the results. We chose to model a diffuse type of fibrosis in which there are no gaps in the myocardium on the scale of a model element: all our elements represented myocardium. Fibrotic elements only had zero transverse conductivity, which was to mimic fine-grained fibrosis that interrupts side-to-side coupling between myofibers [13].

In summary, we have shown that pseudo-periodic AF patterns can be simulated and that this stability can be quantified. A similar quantification of measured results is needed to establish whether the model truly agrees with reality.

\section{Acknowledgments}

We thank Sarah Peris for the implementation of the singularity detection algorithm. This work was supported by the French National Research Agency, grant reference ANR-10-IAHU04-LIRYC. This work was granted access to HPC resources of CINES under GENCI allocation 2019-A0050307379. 


\section{References}

1. Allessie, M.A., de Groot, N., Houben, R.P.M., Schotten, U., Boersma, E., Smeets, J.L., Crijns, H.J.: Electropathological substrate of long-standing persistent atrial fibrillation in patients with structural heart disease: Longitudinal dissociation. Circ. Arrhythm. Electrophysiol. 3, 606-615 (2010)

2. Courtemanche, M., Ramirez, R., Nattel, S.: Ionic mechanisms underlying human atrial action potential properties: insights from a mathematical model. Am. J. Physiol. Heart Circ. Physiol. 275, H301-H321 (1998)

3. Eckmann, J.P., Oliffson Kamphorst, S., Ruelle, D.: Recurrence plots of dynamical systems. Europhys. Lett. 4, 973-977 (1987)

4. Gharaviri, A., Verheule, S., Eckstein, J., Potse, M., Kuklik, P., Kuijpers, N.H.L., Schotten, U.: How disruption of endo-epicardial electrical connections enhances endo-epicardial conduction during atrial fibrillation. Europace 19, 308-316 (2017)

5. Gharaviri, A., Zink, M., Pezzuto, S., Potse, M., Zeemering, S., Krause, R., Auricchio, A., Schotten, U.: Acute changes in P-wave morphology by pulmonary vein isolation in atrial fibrillation patients. In: Computing in Cardiology. Maastricht, The Netherlands (2018)

6. Gray, R.A., Pertsov, A.M., Jalife, J.: Spatial and temporal organization during cardiac fibrillation. Nature 392, 75-78 (1998)

7. Krause, D., Potse, M., Dickopf, T., Krause, R., Auricchio, A., Prinzen, F.W.: Hybrid parallelization of a large-scale heart model. In: Keller, R., Kramer, D., Weiss, J.P. (eds.) Facing the Multicore-Challenge II. Lecture Notes in Computer Science, vol. 7174, pp. 120-132. Springer, Berlin (2012)

8. Lim, H.S., Hocini, M., Dubois, R., Denis, A., Derval, N., Zellerhoff, S., Yamashita, S., Berte, B., Mahida, S., Komatsu, Y., Daly, M., Jesel, L., Pomier, C., Meillet, V., Amraoui, S., Shah, A.J., Cochet, H., Sacher, F., Jaïs, P., Haïssaguerre, M.: Complexity and distribution of drivers in relation to duration of persistent atrial fibrillation. J. Am. Coll. Cardiol. 69, 1257-1269 (2017)

9. Marwan, N., Kurths, J., Foerster, S.: Analysing spatially extended highdimensional dynamics by recurrence plots. Phys. Lett. A 379, 894-900 (2015)

10. Potse, M., Lankveld, T.A.R., Zeemering, S., Dagnelie, P.C., Stehouwer, C.D.A., Henry, R.M., Linnenbank, A.C., Kuijpers, N.H.L., Schotten, U.: P-wave complexity in normal subjects and computer models. J. Electrocardiol. 49, 545-553 (2016)

11. Roney, C.H., Bayer, J.D., Zahid, S., Meo, M., Boyle, P.M.J., Trayanova, N.A., Haïssaguerre, M., Dubois, R., Cochet, H., Vigmond, E.J.: Modelling methodology of atrial fibrosis affects rotor dynamics and electrograms. Europace 18, iv146-155 (2016)

12. Skanes, A.C., Mandapati, R., Berenfeld, O., Davidenko, J., Jalife, J.: Spatiotemporal periodicity during atrial fibrillation in the isolated sheep heart. Circulation 98, 1236-1248 (1998)

13. Spach, M.S., Dolber, P.C.: Relating extracellular potentials and their derivatives to anisotropic propagation at a microscopic level in human cardiac muscle; evidence for electrical uncoupling of side-to-side fiber connections with increasing age. Circ. Res. 58, 356-371 (1986)

14. Zhou, R., Kneller, J., Leon, L.J., Nattel, S.: Development of a computer algorithm for the detection of phase singularities and initial application to analyze simulations of atrial fibrillation. Chaos 12, 764-778 (2002) 